\title{
Exploring the possibilities for star-tracker assisted calibration of the six individual GOCE accelerometers
}

\author{
P. N. A. M. Visser
}

Received: 14 May 2007 / Accepted: 3 December 2007 / Published online: 4 January 2008

(C) The Author(s) 2007

\begin{abstract}
A method has been developed and tested for estimating calibration parameters for the six accelerometers on board the Gravity field and steady-state Ocean Circulation Explorer (GOCE) from star tracker observations. These six accelerometers are part of the gradiometer, which is the prime instrument on board GOCE. It will be shown that by taking appropriate combinations of observations collected by the accelerometers, by modeling acceleration terms caused by gravity gradients from an a priori low-degree spherical harmonic expansion, and by modeling rotational acceleration terms derived from star-tracker observations, scale factors of each of the accelerometers can be estimated for each axis. Simulated observations from a so-called end-to-end simulator were used to test the method. This end-to-end simulator includes a detailed model of the GOCE satellite, its instruments and instrument errors, and its environment. Results of the tests indicate that scale factors of all six accelerometers can be determined with an accuracy of around 0.01 for all components on a daily basis.
\end{abstract}

Keywords GOCE - Gradiometer - Accelerometers · Star-tracker · Calibration - Bias · Bias drift · Scale factor

\section{Introduction}

The first gravity field gradiometer in space will be flown on board of the first European Space Agency (ESA) Earth Explorer mission, the Gravity field and steady-state Ocean

P. N. A. M. Visser ( $\square)$

Delft Institute of Earth Observation and Space Systems (DEOS), Delft University of Technology, Kluyverweg 1,

2629 HS Delft, The Netherlands

e-mail: P.N.A.M.Visser@tudelft.nl
Circulation Explorer (GOCE). The GOCE gradiometer will consist of three orthogonal pairs of accelerometers (Fig. 1). These accelerometers have a sensitivity of the order of $10^{-12} \mathrm{~m} / \mathrm{s}^{2}$. The foreseen launch date of GOCE is in 2008 (as of September 2007). GOCE aims at modeling the static Earth's gravity field with an accuracy of $2 \mathrm{~cm}$ in geoid and $1 \mathrm{mGal}$ in gravity anomaly at a spatial resolution or half-wavelength of $100 \mathrm{~km}$ (Drinkwater et al. 2007), thereby improving the already high-quality static gravity field models that can be derived from observations taken by the GRAvity Climate Experiment (GRACE) mission, whose primary objective is to observe temporal gravity (Tapley et al. 2004).

In addition to the gradiometer, GOCE will be equipped with a dual-frequency GPS receiver, star-trackers, ion engines and magnetic torquers. The satellite gravity gradiometer provides both differential accelerometer observations from which satellite gravity gradients (SGG) and in conjunction rotational accelerations are to be derived, and common-mode (CM) accelerometer observations for representing the nongravitational accelerations and for steering the Drag Free Control (DFC) system. The GPS receiver enables a precise orbit determination and provides especially information for retrieving the low-resolution part of the gravity field, whereas the SGG observations provide high-resolution gravity field information. The ion engines form part of the DFC and reduce the non-gravitational accelerations to a level that prevents saturation of the accelerometers. The magnetic torquers are used for controlling the attitude motion of the satellite.

The highest sensitivity of the gradiometer is in the measurement bandwidth $(\mathrm{MB})$ of $0.005-0.1 \mathrm{~Hz}$. Star tracker observations are required together with differential accelerometer observations to precisely reconstruct the angular motion of the satellite. This is necessary for deriving the SGG observations from the differential accelerations (Alenia 1999). 


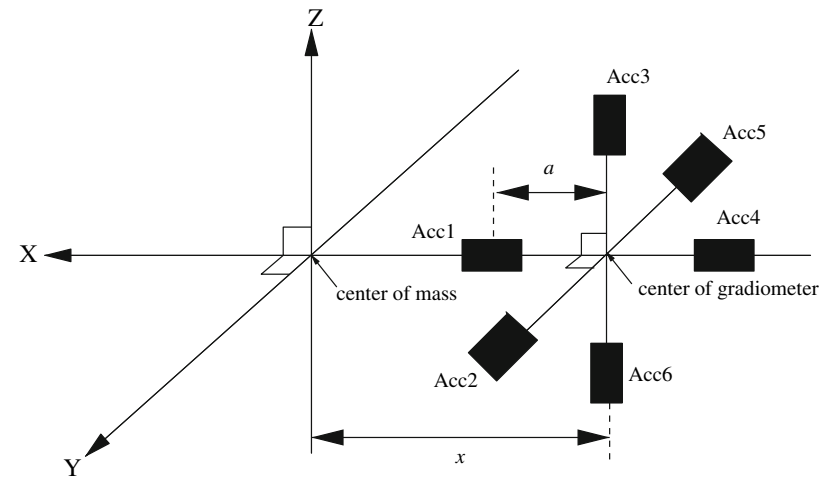

Fig. 1 Configuration and naming convention of the three pairs of accelerometers on orthogonal axes that together form a gravity gradiometer. Half the arm length of each pair is equal to $a$, whereas the offset of the center of the gradiometer with respect to the center of mass of the satellite is indicated by $x$. The $X, Y$ and $Z$ axes are aligned with the along-track (or flight), cross-track and radial (or height) directions, respectively, to within a few degrees

A crucial prerequisite for GOCE mission success is a reliable and high-precision calibration of the gradiometer. A challenging procedure has been developed to calibrate the gradiometer not only pre-launch by a series of on-ground tests, but also after launch by making use of on-board coldgas thrusters to provoke a long series of gradiometer shaking events that will provide observations for its calibration (Cesare and Catastini 2005). The objective is to obtain scale factors for the differential accelerometer observations at a precision level of $10^{-5}$ or better (Cesare 2005). Such a precision level can not be achieved pre-launch due to the $1-\mathrm{g}$ environment.

In addition, a number of post-launch methods has been designed and will be implemented that aim at validating the calibration of the gradiometer instrument and, at the same time, supporting the operations of the satellite (Bouman et al. 2004). These methods are based on (1) comparison with the best available global gravity field models, (2) upward continuation of high-precision ground-based gravity field data over certain geographical areas, and (3) use of GPS highlow satellite-to-satellite tracking (SST) observations (Visser 2007). All these methods make either use of the best available state-of-the art global gravity field models or geographical areas for which high-resolution and high-precision terrestrial gravity field information is available. It will be shown that the method outlined in this paper is not very sensitive to errors in the a priori gravity field model.

For comparison, the CHAMP and GRACE satellites carry only one accelerometer, which is located in the center of mass. The best calibration of these accelerometers is obtained by a precise orbit determination (Bruinsma and Biancale 2003, Bruinsma et al. 2004). For GOCE, the CM can only be calibrated to a limited extend by such a precise orbit determination (Visser 2007).
The focus of this paper will be on an efficient and fast estimation of bias parameters and scale factors of all six individual accelerometers that together form the gradiometer (Fig. 1) by making use of star-tracker observations. The results of this estimation might in reality support and/or validate the other gradiometer calibration methods. First, a detailed model of accelerometer observations will be provided (Sect. 2). This model serves as the basis for the calibration method and for some additional checks (Sect. 3). The method will be applied to a data set of simulated observations generated by a GOCE end-to-end simulator (Sect. 4). Finally, results will be summarized and conclusions will be drawn about the predicted performance of the proposed calibration method (Sect. 5).

\section{Accelerometer observations}

Before introducing methods for calibrating and/or validating the accelerometers on board GOCE, a comprehensive model for their observations is required. First, it is assumed that each individual accelerometer is located away from the center of mass of the satellite with eccentricity vector $\mathbf{x}_{i}{ }^{\mathrm{T}}=(x, y, z)$, where the coordinate axes are in the gradiometer reference frame. The $X$ axis is aligned with the long axis of the satellite, the $Z$ axis is perpendicular to the $X$ axis and is aligned with the wings of the GOCE satellite, and the $Y$ axis completes a right-handed orthonormal frame (see also Fig. 1).

For a perfectly Earth nadir pointing satellite, the $X, Y$ and $Z$ axes would coincide with the along-track, cross-track and radial directions, respectively. In reality, the GOCE satellite attitude will be steered in order to minimize aerodynamic torques ("head in the wind") resulting in yaw, roll and pitch angles of the order of a few degrees (Visser 2007). The axes of the gradiometer reference frame are defined to nominally be aligned with the axes of the accelerometers (in reality there will be small misalignments, see below).

Second, it is assumed that each accelerometer is affected by biases, scale factors and misalignments. In reality, the accelerometers will also be affected by non-orthogonalities, couplings and quadratic terms (Cesare and Catastini 2005). However, the requirement for the combined effect of nonorthogonalities of, and couplings between the accelerometer axes is smaller than $1.3 \times 10^{-4} \mathrm{rad}$. The residual quadratic effects are assumed to be negligible. In that case, the accelerations observed by the $i$-th accelerometer $(i=1, \ldots, 6)$ can be written as:

$\mathbf{a}_{\mathrm{obs}, i}=\mathbf{S}_{i}\left[\mathbf{M}_{\mathbf{i}}(\boldsymbol{\Gamma}+\mathbf{R}) \mathbf{x}_{i}+\mathbf{d}\right]+\mathbf{b}_{i}+\epsilon_{i}$.

The (diagonal) matrix of accelerometer scale factors $S_{i, k}$ $(k=x, y, z)$ is represented by $\mathbf{S}_{i}$ : 
$\mathbf{S}_{i}=\left(\begin{array}{ccc}S_{i, x} & 0 & 0 \\ 0 & S_{i, y} & 0 \\ 0 & 0 & S_{i, z}\end{array}\right)$.

$\mathbf{M}$ represents the misalignment matrix:

$\mathbf{M}_{\mathbf{i}}=\left(\begin{array}{rrr}1 & -\gamma_{i} & \beta_{i} \\ \gamma_{i} & 1 & -\alpha_{i} \\ -\beta_{i} & \alpha_{i} & 1\end{array}\right)$.

The misalignments are due to instrument imperfections and are represented by rotations around the individual accelerometer axes, where $\alpha_{i}, \beta_{i}$ and $\gamma_{i}$ are the roll, pitch and yaw angles. It is assumed that these angles are very small, leading to the linearized matrix in Eq. (3). In the following, the misalignments are nominally ignored. Tests have indicated that for the simulated data used for this paper, the misalignments are indeed small (Sect. 4).

$\boldsymbol{\Gamma}$ is the gravity gradient matrix containing the secondorder derivatives of the gravitational field potential $\Gamma_{k l}(k, l=$ $x, y, z)$ at the satellite location:

$\boldsymbol{\Gamma}=\left(\begin{array}{ccc}\Gamma_{x x} & \Gamma_{x y} & \Gamma_{x z} \\ \Gamma_{y x} & \Gamma_{y y} & \Gamma_{y z} \\ \Gamma_{z x} & \Gamma_{z y} & \Gamma_{z z}\end{array}\right)$.

The matrix with rotational terms $\mathbf{R}$ is written as:

$$
\begin{aligned}
\mathbf{R}= & \left(\begin{array}{ccc}
r_{x x} & r_{x y} & r_{x z} \\
r_{y x} & r_{y y} & r_{y z} \\
r_{z x} & r_{z y} & r_{z z}
\end{array}\right) \\
= & \left(\begin{array}{ccc}
-\omega_{y}^{2}-\omega_{z}^{2} & \omega_{y} \omega_{x} & \omega_{z} \omega_{x} \\
\omega_{x} \omega_{y} & -\omega_{x}^{2}-\omega_{z}^{2} & \omega_{z} \omega_{y} \\
\omega_{x} \omega_{z} & \omega_{y} \omega_{z} & -\omega_{x}^{2}-\omega_{y}^{2}
\end{array}\right) \\
& +\left(\begin{array}{ccc}
0 & -\dot{\omega}_{z} & \dot{\omega}_{y} \\
\dot{\omega}_{z} & 0 & -\dot{\omega}_{x} \\
-\dot{\omega}_{y} & \dot{\omega}_{x} & 0
\end{array}\right),
\end{aligned}
$$

where $\omega_{k}$ and $\dot{\omega}_{k}$ represent the angular rotation rates $\omega^{\mathrm{T}}=$ $\left(\omega_{x}, \omega_{y}, \omega_{z}\right)$ and the angular acceleration rates $\dot{\omega}^{\mathrm{T}}=$ $\left(\dot{\omega}_{x}, \dot{\omega}_{y}, \dot{\omega}_{z}\right)$. For the calibration method that is the topic of this paper, the elements of the matrix with rotational terms can be derived from the star-tracker observations by single and double differentiation in time of observed orientation angles (Sect. 3).

Finally, $\mathbf{d}^{\mathrm{T}}=\left(d_{x}, d_{y}, d_{z}\right), \mathbf{b}_{i}{ }^{\mathrm{T}}=\left(b_{i, x}, b_{i, y}, b_{i, z}\right)$, and $\epsilon_{i}^{\mathrm{T}}=\left(\epsilon_{i, x}, \epsilon_{i, y}, \epsilon_{i, z}\right)$ represent the non-gravitational accelerations, the accelerometer biases, and the observation errors for the three accelerometer axes.

\section{Methodology}

The calibration method and some checks that will be outlined in this Section are based on the assumption that each individual accelerometer experiences the same linear non-gravitational accelerations $\mathbf{d}$ (accelerations induced by torques are included in the rotational terms):

$\mathbf{d}=\mathbf{S}_{i}^{-1}\left(\mathbf{a}_{\mathrm{obs}, i}-\mathbf{b}_{i}-\epsilon_{i}\right)-(\boldsymbol{\Gamma}+\mathbf{R}) \mathbf{x}_{\mathbf{i}}$.

By imposing that the non-gravitational accelerations are the same for each individual accelerometer, each accelerometer can be scaled with respect to the others in conjunction with the estimation of relative accelerometer biases (Sect. 4). Before outlining the calibration method by star-tracker observations, the different contributions to the total accelerometer observations (Eq. (1)) are assessed.

\subsection{Correcting the accelerometer observations}

Each accelerometer experiences different accelerations due to the local satellite gravity gradient $\left(\boldsymbol{\Gamma} \mathbf{x}_{i}\right.$ in Eq. (1)) and due to rotational effects (angular accelerations and centrifugal terms, $\mathbf{R} \mathbf{x}_{i}$ in Eq. (1)). First of all, the location of each individual accelerometer has to be defined:

$\mathbf{x}_{\mathbf{1}}^{\mathrm{T}}=\left(o_{x}+L_{x} / 2, o_{y}, o_{z}\right)$

$\mathbf{x}_{\mathbf{2}}^{\mathrm{T}}=\left(o_{x}, o_{y}+L_{y} / 2, o_{z}\right)$

$\mathbf{x}^{\mathrm{T}}=\left(o_{x}, o_{y}, o_{z}+L_{z} / 2\right)$

$\mathbf{x}_{4}^{\mathrm{T}}=\left(o_{x}-L_{x} / 2, o_{y}, o_{z}\right)$

$\mathbf{x}_{5}{ }^{\mathrm{T}}=\left(o_{x}, o_{y}-L_{y} / 2, o_{z}\right)$

$\mathbf{x}_{\mathbf{6}}{ }^{\mathrm{T}}=\left(o_{x}, o_{y}, o_{z}-L_{z} / 2\right)$,

where, $L_{x}, L_{y}, L_{z}$ are the gradiometer arm lengths along the $X, Y$ and $Z$ axes (nominally $50 \mathrm{~cm}$ for the end-to-end simulator, in Cesare and Catastini (2005) the arm length along the $X$ axis slightly deviates and is equal to $51.4 \mathrm{~cm}$ ), and $\mathbf{o}^{\mathrm{T}}=\left(o_{x}, o_{y}, o_{z}\right)$ represents the offset of the center of the gradiometer instrument with respect to the satellite center of mass (of the order of a few $\mathrm{cm}$ for the end-to-end simulated data).

Second, the gravity gradients can be derived from an a priori gravity field model. It will be shown that for the calibration method outlined in Sect. 3.2, a low degree and order gravity field model, for example truncated at degree and order 20, suffices. Finally, rotational terms need to be accounted for. The star-tracker observations provide the orientation of the gradiometer reference frame in the J2000 inertial reference frame (Montenbruck and Gill 2000). They are used, together with orbital information (position and velocity) to derive the yaw, pitch and roll angles $\left(\phi_{i}, i=1,2,3\right)$ of the axes of the gradiometer reference frame with respect to the radial, along-track and cross-track directions (Visser 2007).

It has to be noted that the axes of the selected star-tracker are not aligned with those of the gradiometer reference frame. In fact, a rotation of $60^{\circ}$ around the $X$ axis has to be applied to the star-tracker observations. The relatively large star-tracker observations errors around the bore sight (Visser 2007) are 
thus spread over more axes. This means that the results presented in this paper might be considered pessimistic. In reality, it might be possible to combine observations taken by different star-trackers. GOCE will carry three star-trackers, with two always observing. These star-trackers have different orientations, thereby opening the possibility to reduce the effect of the large errors around the bore sight direction of the currently selected one star tracker.

First and second time derivatives of the rotation angles are obtained by using a moving time window of certain width over the time-series of these angles and fitting second-order polynomials:

$\phi_{i}=a_{0}+a_{1} t+\frac{1}{2} a_{2} t^{2}$,

where $t$ represents time, the coefficient $a_{0}$ the orientation angle, and $a_{1}$ and $a_{2}$ represent the angular rotation rate and acceleration, respectively. Depending on the noise characteristics of the star-tracker, a longer or shorter time window might be required. The end-to-end simulator data were used to determine the length of the window (see below).

In all cases to be described in this paper, use has been made of one day of simulated GOCE observations (11 April 2008 , similar results were found for other days) from the end-to-end simulator (Catastini et al. 2007, Visser 2007). The following accelerometer error sources were taken into account by this end-to-end simulator: frequency dependent accelerometer observation noise, quadratic terms, couplings, misalignments of the axes of the individual accelerometers, biases, bias drifts and scale factor values. In addition, the star-tracker observations were corrupted by realistic measurement errors. Moreover, the satellite environment is modeled in great detail, including the full EGM96 gravity field model (Lemoine et al. 1998) and non-gravitational forces. The thrusting and actuation by the DFC and magnetic torquers were taken ito account as well. The star-tracker observation errors are typically of the order of $10^{\prime \prime}$ ( $\operatorname{arcsec}$ ) for the more precise rotation axes and up to $50^{\prime \prime}$ for the bore sight axis (Visser 2007) and are provided with a $0.5 \mathrm{~s}$ time interval ( $1 \mathrm{~s}$ for the accelerometer observations). A time window of $100 \mathrm{~s}$ was found by trial and error to lead to small errors of derived angular rotation rates and accelerations for all three axes (Eq. (8)). In fact, by comparison with the known values provided as part of the end-to-end data set, the angular acceleration errors were found to be of the order of $1 \times 10^{-8} \mathrm{rad} / \mathrm{s}^{2}$ around the $Y$ and $Z$ axes (pitch and yaw) and $3 \times 10^{-8} \mathrm{rad} / \mathrm{s}^{2}$ around the $X$ axis (roll). It has to be noted that the accelerometer scale factor deviations from one that have been applied to the simulated data are less than $0.001(0.1 \%)$, which is thus the accuracy level to which the proposed method can be tested.

In reality, the true angular acceleration values are not known. The question might therefore be raised how to determine the value for the length of the time window (Eq. (8)). One possibility is to use values that are based on the predicted behavior of the entire GOCE satellite as modeled by the end-to-end simulator (as was done in this paper). After launch of GOCE, the actual performance of all instruments (such as noise characteristics) will be carefully assessed and this might then be used for a new end-to-end simulator run possibly resulting in updates of the time window length.

Time-series of observations for accelerometers 1 and 2 are displayed in Fig. 2 after reducing for the full $360 \times 360$ EGM96 gravity gradients and rotational terms derived from the star-tracker observations, and after subtracting the mean of the reduced observations. Already a close match can be observed. Differences between the observations of accelerometers 1 and 2 are displayed in Fig. 3, before and after reducing for gravity gradients and rotational terms.

It can be observed in Fig. 3 that a close match between the individual accelerometers is obtained after correcting for both gravity gradient and rotational terms, apart from systematic effects. It can now be seen that the accelerometers do not only suffer from biases, but also drifts (as part of the colored noise). Therefore, the accelerometer observation model (Eq. (1)) has been optionally extended by bias drift factors as well, or:

$b_{i}=b_{i, 0}+b_{i, t} t$

where, $b_{i, 0}$ and $b_{i, t}$ represent the bias at the starting epoch and the bias drift, respectively.

\subsection{Validation by star-tracker observations}

By imposing that each accelerometer observes the same nongravitational accelerations and by comparing accelerometer $i$ with accelerometer $j$, Eq. (6) leads to:

$$
\begin{aligned}
& \mathbf{S}_{\mathbf{i}}^{-1}\left(\mathbf{a}_{\mathrm{obs}, i}-\mathbf{b}_{i}-\epsilon_{i}\right)-\mathbf{S}_{\mathbf{j}}^{-1}\left(\mathbf{a}_{\mathrm{obs}, j}-\mathbf{b}_{j}-\epsilon_{j}\right) \\
& \quad=(\boldsymbol{\Gamma}+\mathbf{R})\left(\mathbf{x}_{\mathbf{i}}-\mathbf{x}_{\mathbf{j}}\right) .
\end{aligned}
$$

By using an a priori model for the gravity gradients, deriving the rotational terms from the star-tracker observations and assuming that the observation noise is negligible, Eq. (10) can be solved for the accelerometer biases and scale factors. Please note that for each axis a singularity arises, namely one bias value. Therefore, one accelerometer needs to be defined as being the so-called reference accelerometer, which means that all biases will be relative to the biases of this accelerometer. As an example, the following observation equation can be derived for the $X$-axis component (indicated by $x$ ) of the first and second accelerometers (applying Eq. (7)): 

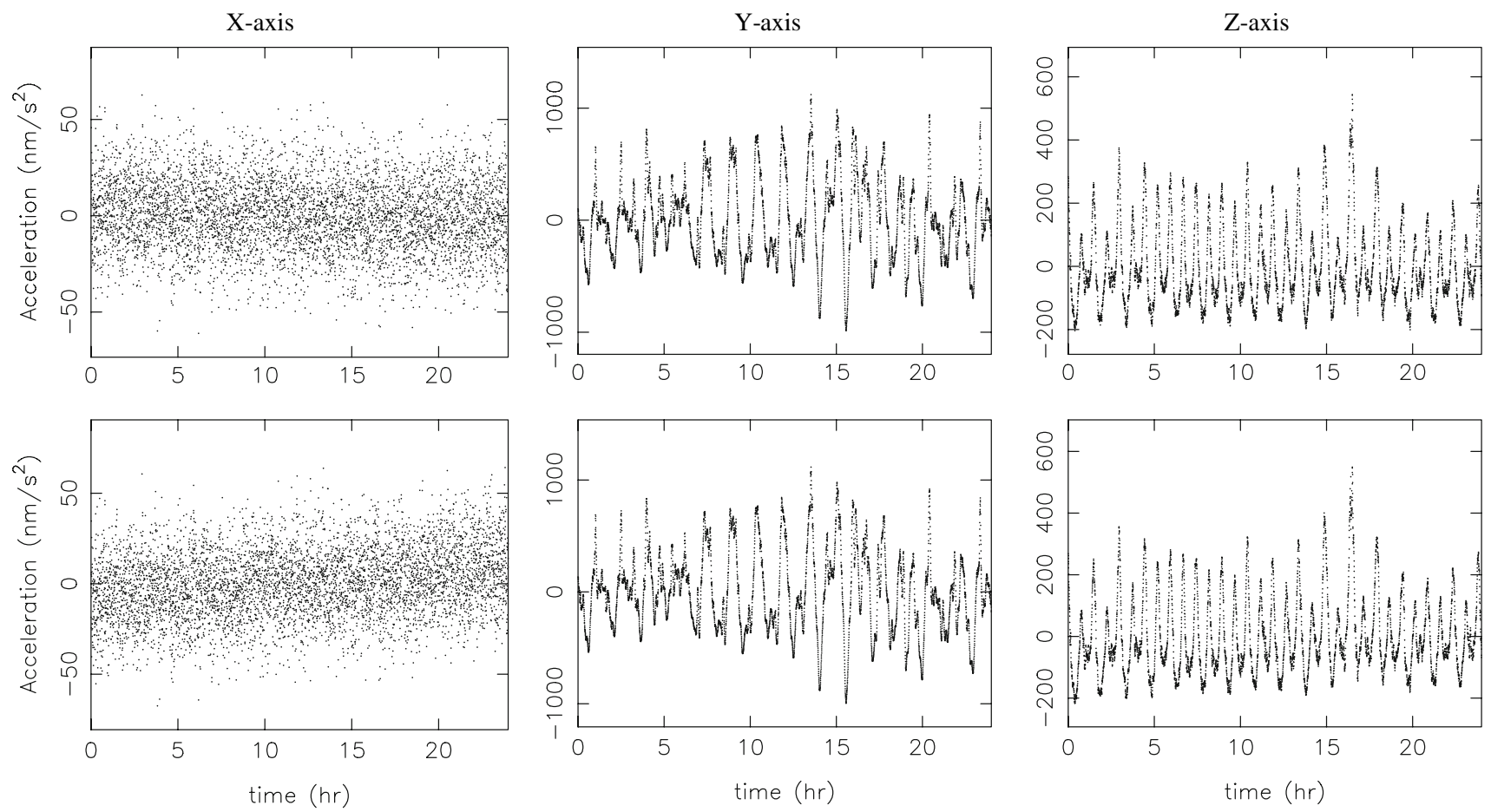

Fig. 2 Observations of accelerometers 1 (top) and 2 (bottom) in the gradiometer reference frame after correcting for both rotational and gravity gradient terms, and after taking away the mean. Note that the signal along the $X$ axis is relatively small and displays a noisy behavior

$$
\begin{aligned}
& \frac{a_{\mathrm{obs}, 1, x}-b_{1, x}}{S_{1, x}}-\frac{a_{\mathrm{obs}, 2, x}-b_{2, x}}{S_{2, x}} \\
& =\left(r_{x x}+\Gamma_{x x}\right) \frac{L_{x}}{2}-\left(r_{x y}+\Gamma_{x y}\right) \frac{L_{y}}{2} .
\end{aligned}
$$

In this case, only the relative bias $b_{2, x}-b_{1, x}$ can be determined. Eq. (11) is also an example of an equation that can be used to estimate the arm lengths of the gradiometer (Sect. 4). In fact, the inverse of the scale factors $\left(S_{i, k}{ }^{-1}\right)$ is estimated and the relative biases divided by the scale factors $\left(\left(b_{2, x}-\right.\right.$ $\left.b_{1, x}\right) / S_{i, k}$ ) in order to have observation equations that are linear for the estimated parameters. The actual values for the scale factors and relative biases (and possibly also formal errors, see below) can then easily be derived from these estimated parameters.

In all cases, the observation equations are solved by the method of unweighted least-squares, i.e. each accelerometer observation is assigned the same weight. In addition, the estimation is regularization-free and thus unbiased. It might be argued that uniform weighting is sub-optimal considering the anticipated colored noise behavior of the accelerometers, the different noise level for the star tracker bore sight and other directions, and also considering that each accelerometer will have two sensitive and one less-sensitive axes (ESA 1999). It is anticipated that the accelerometers will have the highest sensitivity in the MB $(0.005-0.1 \mathrm{~Hz})$, but it has to be realized that the non-gravitational accelerations will be dominant at the low frequencies (see e.g. Fig. 2). Thus, applying for example a frequency-dependent weighting commensurate with the noise behavior would on the one hand reduce the negative impact of low-frequency accelerometer observation noise, but on the other hand significantly degrade the signal-to-noise ratio (i.e. only a small non-gravitational signal is then left for calibration). Not having to rely on prior knowledge about the observation error characteristics (which might in reality turn out to be different) makes the method more robust/rigorous, and can be considered as an advantage. In addition, it has to be realized that applying a bandpass filter to the observation equations will lead to unobservability of, for example, accelerometer biases. Therefore, frequencydependent weighting has not been investigated for the current implementation of the method.

It has to be noted that all calibration parameters are estimated simultaneously, whereas the uniform weighting in fact leads to a situation where parameters for different axes are uncorrelated. Thus the same results would be obtained by having three separate estimations of calibration parameters, one for each axis. This would be computationally more efficient. However, the computations are not computationally demanding (typically less than one minute CPU time on a $3 \mathrm{GHz}$ Linux PC). Moreover, the current implementation would allow the inclusion of frequency dependent weighting 

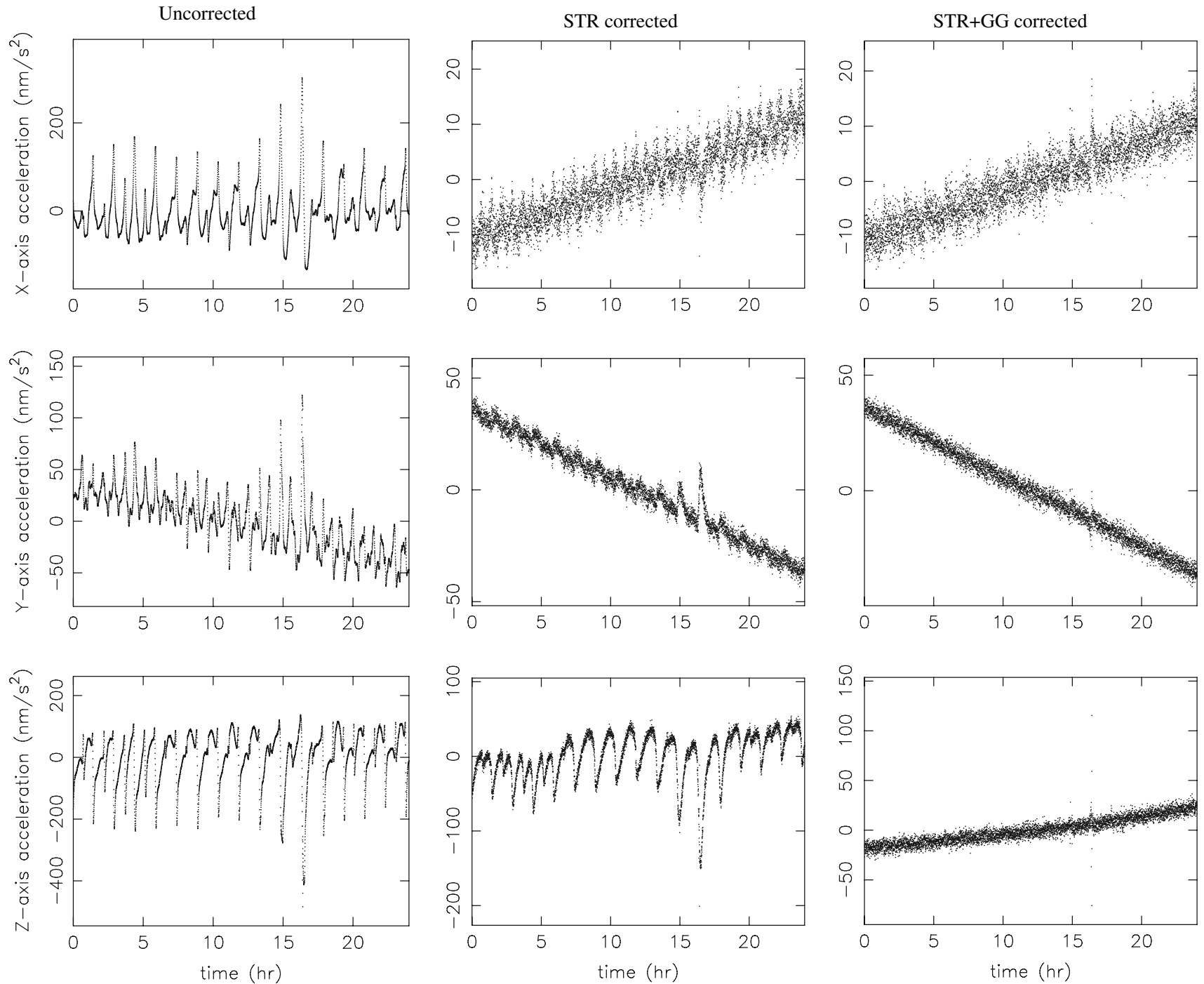

Fig. 3 Difference between observations of accelerometers 1 and 2 before corrections (left), after correcting for rotation terms derived from the star-tracker observations (middle), after applying gravity gradient corrections (right) in the gradiometer reference frame, and after taking

and correlated observation errors between different axes if this is found to be valuable in the future.

\section{Results}

A least-squares estimation of accelerometer biases, bias drifts (optionally) and scale factors has been conducted using one day (11 April 2008) of simulated end-to-end star-tracker and accelerometer observations. In addition, orbital information was included in the end-to-end data package, which allowed for the computation of gravity gradients. The orbital information includes rotation parameters connecting the $\mathrm{J} 2000$ and Earth-fixed reference frames. Together with the star-tracker away the mean. "STR" indicates that the accelerometer observations are corrected for rotational terms derived from the star-tracker, and "GG" corrected for gravity gradient terms

observations, this enabled the computation of gravity gradients in the gradiometer reference frame.

\subsection{Verification of assumptions}

A number of tests was conducted first to verify some of the assumptions that have been made. First, relative scale factors were estimated. By imposing that each accelerometer experiences the same non-gravitational accelerations, the following equation can be derived from Eqs. 6 and 10:

$$
\begin{aligned}
& \mathbf{S}_{i}^{-1}\left(\mathbf{a}_{\mathrm{obs}, i}-\mathbf{b}_{i}-\epsilon_{i}\right)-(\boldsymbol{\Gamma}+\mathbf{R}) \mathbf{x}_{\mathbf{i}} \\
& \quad=\mathbf{S}_{j}^{-1}\left(\mathbf{a}_{\mathrm{obs}, j}-\mathbf{b}_{j}-\epsilon_{j}\right)-(\boldsymbol{\Gamma}+\mathbf{R}) \mathbf{x}_{\mathbf{j}} .
\end{aligned}
$$


By taking into account the rotational and gravity gradient terms, relative scale factors $\left(S_{i, k} / S_{j, k}\right)$ and biases $\left(b_{i}-b_{j}\right)$ were estimated for every possible pair of accelerometers. It was found that relative scale factors could deviate from 1 by as much as 0.18 for the $X$ components when bias drifts are not estimated. When these drifts are included in the estimation process, the relative scale factors deviate from 1 by less than 0.001 for the $Y$ and $Z$ axes. The scale factors for the $X$ direction can still deviate from 1 by more than 0.10 , but the mean value does not deviate from 1 significantly. It has to be noted that the drag-free control especially compensates the non-gravitational accelerations in the $X$ direction (Fig. 2) reducing the accelerations in this direction to a noisy signal with a relatively small variance. For such a signal, the estimation of scale factors becomes less reliable (a large signal with systematic features is easier to scale). This result indicates that a frequency dependent weighting where the low frequencies are assigned a low weight, most likely results in a reduced observability of accelerometer biases and scale factors. The obtained results do not contradict the assumption that the end-to-end simulated accelerometer observations have scale factors that deviate from 1 by more than 0.001 .

Second, upper bounds for the misalignments were estimated. By assuming that the scale factors are close to one ( $\mathbf{S}=\mathbf{I})$, the following equation can be derived from Eq. (1):

$$
\begin{aligned}
& \mathbf{M}_{\mathbf{i}}^{-1}\left(\mathbf{a}_{\mathrm{obs}, i}-\mathbf{b}_{i}-\epsilon_{i}\right)-\mathbf{M}_{\mathbf{j}}^{-1}\left(\mathbf{a}_{\mathrm{obs}, j}-\mathbf{b}_{j}-\epsilon_{j}\right) \\
& \quad=(\boldsymbol{\Gamma}+\mathbf{R})\left(\mathbf{x}_{\mathbf{i}}-\mathbf{x}_{\mathbf{j}}\right) .
\end{aligned}
$$

Again, by using an a priori gravity field model for computing the gravity gradients $\boldsymbol{\Gamma}$ and the star-trackers to derive rotational terms, this time the misalignments $\alpha_{i}, \beta_{i}, \gamma_{i}$ can be estimated together with relative bias values $b_{i}-b_{j}$. The first accelerometer was used as reference for the relative bias estimates. The upper bound of the misalignments was found to be below $0.01 \mathrm{rad}$. It has to be noted that the estimated misalignments include the deviation of the accelerometer scale factors from one. In fact, the values represent the product of $\mathbf{S}$ and $\mathbf{M}$ and thus implicitly also reflect the value or uncertainty of the accelerometer scale factors.

Finally, by assuming that the scale factors are close to 1 , Eqs. 10 and 7 can be used to estimate the arm lengths of the three orthogonal pairs of accelerometers:

$$
\begin{aligned}
& \left(\mathbf{a}_{\mathrm{obs}, i}-\mathbf{b}_{i}-\epsilon_{i}\right)-\left(\mathbf{a}_{\mathrm{obs}, j}-\mathbf{b}_{j}-\epsilon_{j}\right) \\
& \quad=(\boldsymbol{\Gamma}+\mathbf{R})\left(\mathbf{x}_{\mathbf{i}}-\mathbf{x}_{\mathbf{j}}\right) .
\end{aligned}
$$

By using an a priori gravity field model for computing the gravity gradients $\Gamma$ and the star-trackers to derive rotational terms, the values for $L_{x}, L_{y}$ and $L_{z}$ were found to be close to $50 \mathrm{~cm}$, which was the value used for all arms in the endto-end simulation. The maximum deviation was found to be less than $0.4 \mathrm{~cm}$.

\begin{tabular}{|c|c|c|c|}
\hline Acc. & SF X & SF Y & SF Z \\
\hline \multicolumn{4}{|c|}{ No bias drifts } \\
\hline 1 & 0.966 & 0.751 & 0.977 \\
\hline 2 & 0.976 & 0.742 & 0.967 \\
\hline 3 & 0.898 & 0.737 & 0.973 \\
\hline 4 & 0.982 & 0.737 & 0.969 \\
\hline 5 & 0.987 & 0.743 & 0.977 \\
\hline 6 & 0.972 & 0.751 & 0.972 \\
\hline \multicolumn{4}{|c|}{ Including bias drifts } \\
\hline 1 & 0.994 & 0.977 & 0.993 \\
\hline 2 & 0.991 & 0.977 & 0.992 \\
\hline 3 & 0.989 & 0.976 & 0.992 \\
\hline 4 & 0.996 & 0.976 & 0.989 \\
\hline 5 & 0.992 & 0.977 & 0.992 \\
\hline 6 & 0.992 & 0.978 & 0.991 \\
\hline \multicolumn{4}{|c|}{ Including bias drifts \& $100 \mathrm{~s}$ averaging } \\
\hline 1 & 1.004 & 0.990 & 1.007 \\
\hline 2 & 0.993 & 0.989 & 1.007 \\
\hline 3 & 1.019 & 0.989 & 1.007 \\
\hline 4 & 1.002 & 0.989 & 1.006 \\
\hline 5 & 0.993 & 0.989 & 1.005 \\
\hline 6 & 1.013 & 0.991 & 1.005 \\
\hline \multicolumn{4}{|c|}{ Including bias drifts $\& 25 \mathrm{~s}$ window + averaging } \\
\hline 1 & 1.001 & 0.998 & 0.982 \\
\hline 2 & 0.984 & 0.999 & 0.979 \\
\hline 3 & 0.973 & 1.000 & 0.972 \\
\hline 4 & 0.999 & 1.000 & 0.958 \\
\hline 5 & 0.985 & 0.999 & 0.977 \\
\hline 6 & 0.962 & 0.999 & 0.974 \\
\hline
\end{tabular}

Table 1 Estimated scale factors for the individual accelerometers using the star-tracker observations nominally using an averaging window of $100 \mathrm{~s}$

In certain cases, bias drifts have been estimated for all axes of the individual accelerometers. In all cases, the first accelerometer served as reference and a truncated $20 \times 20$ EGM96 gravity field model was used. Also, results are included for the case where the observation equations were averaged over the same time interval as used for the polynomials that represent the angular motion (Eq. (8))

$S F$ scale factor

\subsection{Calibration by star-tracker observations}

The calibration method as outlined in Sect. 3.2 was applied to the selected one day of simulated end-to-end observations as well. The estimated scale factors are included in Table 1 for different combinations of estimated parameters (i.e. inclusion or exclusion of bias drifts), and also for the case where the effect of the estimation window applied to the star-tracker observations (Eq. (8)) was taken into account in the observation equations (Eq. (10)). The estimation window was taken equal to $100 \mathrm{~s}$, which results in angular and centrifugal acceleration terms that can be considered as averages 
over this window. Therefore, the possibility to average the observation equations (Eq. (10)) over this window was built in as well.

The values for the estimated relative biases and bias drifts are in the range of $10^{-8}$ to $10^{-7} \mathrm{~m} / \mathrm{s}^{2}$ and less than $10^{-12} \mathrm{~m} / \mathrm{s}^{3}$, respectively. Accelerometer bias and bias drift values are not very critical for the GOCE gravity field mission performance considering the bandwidth requirement $(0.005-$ $0.1 \mathrm{~Hz}$ ). For example, a constant bias can be considered as an error with a frequency of $0 \mathrm{~Hz}$ and is thus outside the gradiometer measurement bandwidth that will be used for the gravity field recovery. It was found that the magnitude of the simulated accelerometer bias drifts necessitate their inclusion in the estimation process. The scale factors improve considerably, i.e. are much closer to 1 , when co-estimating accelerometer bias drifts. Especially for the $Y$ direction the scale factors improve from about 0.75 (25\% error) to 0.98 ( $2 \%$ error, Table 1). Relatively large bias drifts were found for the $Y$ direction, which can be attributed to the configuration of sensitive and less-sensitive axes of the individual accelerometers (ESA 1999, Cesare 2005).

A significant improvement is also obtained for the $Y$ direction when taking into account the averaging window used for estimating the star-tracker polynomials. In the $Y$ direction, relatively sharp changes in the rotational accelerations are due to the yaw motion, causing relatively large modeling errors when the averaging is not taken into account. All scale factors now typically deviate less than $0.01-0.02$ from 1 , all scale factors for the $Y$ direction being a bit too small, and for the $Z$ direction being a bit too big.

These small systematic deviations can probably still be explained by small modeling errors, since the contribution of the centrifugal accelerations is in fact not based on the average over the $100 \mathrm{~s}$ window of these accelerations but on the average of the angular rate (which is then e.g. squared for the diagonal matrix elements, Eq. (5)). It has to be noted as well that these systematic deviations are different for the $X, Y$ and $Z$ axis. It has to be realized that the satellite will experience different accelerations along the different axes, resulting in different averaging errors. It might therefore be argued that improvements can be obtained by using different averaging windows for different axes. For example, best results for the $X, Y$ and $Z$ axes are obtained with averaging window close to 100,25 and 100 s, respectively.

However, it has been shown that the proposed method of estimating scale factors by star-tracker observations is feasible and can serve as a valuable addition to other proposed methods, such as described in Bouman et al. (2004).

Both the full $360 \times 360$ (i.e. the "real-world" model) and a truncated $20 \times 20$ version of the EGM96 gravity field model were used as a priori model for computing the gravity gradients. In addition, also estimations were done with the JGM3 gravity field model (Tapley et al. 1996). This was done in
Table 2 Estimated scale factors for the individual accelerometers using the star-tracker observations and using an averaging window of $100 \mathrm{~s}$. In all cases, bias drifts have been estimated for all axes of the individual accelerometers and the averaging window was applied to the accelerometer observations as well. Different gravity field modeling has been used. The first accelerometer was used as reference

\begin{tabular}{|c|c|c|c|}
\hline Acc. & SF X & SF Y & SF Z \\
\hline \multicolumn{4}{|c|}{ EGM96, central term only } \\
\hline 1 & 0.949 & 0.991 & 1.001 \\
\hline 2 & 0.991 & 0.990 & 1.008 \\
\hline 3 & 1.020 & 0.990 & 0.999 \\
\hline 4 & 0.942 & 0.990 & 1.013 \\
\hline 5 & 0.999 & 0.991 & 1.006 \\
\hline 6 & 1.016 & 0.992 & 1.014 \\
\hline \multicolumn{4}{|c|}{ EGM96, $20 \times 20$} \\
\hline 1 & 1.004 & 0.990 & 1.007 \\
\hline 2 & 0.993 & 0.989 & 1.007 \\
\hline 3 & 1.019 & 0.989 & 1.007 \\
\hline 4 & 1.002 & 0.989 & 1.006 \\
\hline 5 & 0.993 & 0.989 & 1.005 \\
\hline 6 & 1.013 & 0.991 & 1.005 \\
\hline \multicolumn{4}{|c|}{ EGM96, $360 \times 360$} \\
\hline 1 & 1.004 & 0.990 & 1.007 \\
\hline 2 & 0.993 & 0.989 & 1.007 \\
\hline 3 & 1.019 & 0.989 & 1.007 \\
\hline 4 & 1.002 & 0.989 & 1.006 \\
\hline 5 & 0.993 & 0.989 & 1.005 \\
\hline 6 & 1.013 & 0.991 & 1.005 \\
\hline \multicolumn{4}{|c|}{ JGM3, $70 \times 70$} \\
\hline 1 & 1.004 & 0.990 & 1.007 \\
\hline 2 & 0.993 & 0.989 & 1.007 \\
\hline 3 & 1.019 & 0.989 & 1.007 \\
\hline 4 & 1.002 & 0.989 & 1.006 \\
\hline 5 & 0.993 & 0.989 & 1.005 \\
\hline 6 & 1.013 & 0.991 & 1.005 \\
\hline
\end{tabular}

$S F$ scale factor

order to study the sensitivity of the calibration method to a priori gravity field model error. Scale factor differences are typically less than 0.001 (Table 2), except when only the central term is used. Even in the latter case, the scale factor estimates change generally by at most 0.01 (except for one scale factor value of about 0.94 ) showing that using a state-ofthe-art gravity field model will be more than adequate for the proposed calibration method. In fact, the contribution to the accelerometer observation of errors in the gravity gradient terms due to gravity field model uncertainty is much smaller than the end-to-end predicted non-gravitational accelerations and the accelerations caused by rotational effects (an indication for this is already given by Fig. 3). 
Table 3 Estimated scale factors for the individual accelerometers using the orientation angles derived from the differential accelerometer observations themselves (optimally combined with the star-tracker observations). In all cases, bias drifts have been estimated for all axes of the individual accelerometers and the averaging window was applied to the accelerometer observations as well. The total $360 \times 360$ EGM96 model was selected (i.e. no gravity field model error). The first accelerometer was used as reference

\begin{tabular}{|c|c|c|c|}
\hline Acc. & SF X & SF Y & SF Z \\
\hline \multicolumn{4}{|c|}{$10 \mathrm{~s}$ window \& averaging } \\
\hline 1 & 1.001 & 0.927 & 1.025 \\
\hline 2 & 0.942 & 0.924 & 1.029 \\
\hline 3 & 1.005 & 0.924 & 1.029 \\
\hline 4 & 1.001 & 0.921 & 1.031 \\
\hline 5 & 0.942 & 0.924 & 1.034 \\
\hline 6 & 1.003 & 0.925 & 1.027 \\
\hline \multicolumn{4}{|c|}{$25 \mathrm{~s}$ window \& averaging } \\
\hline 1 & 1.001 & 0.992 & 1.000 \\
\hline 2 & 0.999 & 0.992 & 1.001 \\
\hline 3 & 0.999 & 0.992 & 1.000 \\
\hline 4 & 1.001 & 0.992 & 1.000 \\
\hline 5 & 1.000 & 0.992 & 1.000 \\
\hline 6 & 1.004 & 0.993 & 1.000 \\
\hline \multicolumn{4}{|c|}{$100 \mathrm{~s}$ window \& averaging } \\
\hline 1 & 1.005 & 0.983 & 1.010 \\
\hline 2 & 0.993 & 0.982 & 1.012 \\
\hline 3 & 1.018 & 0.982 & 1.011 \\
\hline 4 & 1.004 & 0.981 & 1.012 \\
\hline 5 & 0.994 & 0.982 & 1.009 \\
\hline 6 & 1.015 & 0.983 & 1.010 \\
\hline
\end{tabular}

$S F$ scale factor

In order to assess the effect of star-tracker observation errors, the method was also tested with quaternions from the end-to-end simulator that were derived from the gradiometer differential accelerometer observations. By taking appropriate combinations of these differential accelerometer observations, angular accelerations can be obtained that are integrated in time and merged optimally through a Kalman filter in order to obtain orientation angles with a very low noise level (Cesare and Catastini 2005). Almost per definition, these orientation angles and their time evolution should match perfectly with the angular and centrifugal accelerations contributions to the total accelerometer observations. As can be seen in Table 3, all the scale factor deviations from 1 are now significantly below 0.01 for an averaging window of $25 \mathrm{~s}$.

Statistical information about the estimated parameters can be retrieved from the estimation process itself. The unweighted least-squares estimation results in invertible normal equation systems. When estimating accelerometer scale factor parameters in conjunction with both relative biases and bias drifts, the minimum and maximum correlation between the estimated parameters is equal to -0.8793 and 0.9851 , respectively (obtained from the inverse of the least-squares normal matrix). In fact, for each axis of each accelerometer a correlation of about -0.87 was found between the bias and bias drift ( 15 combinations). The relatively high correlations of around $0.97-0.98$ were found between all scale factor parameters for the $Y$ axes of the accelerometers, which is due to the relatively large common signal in this direction caused by the yaw motion (see also Fig. 2). The normal equation system is built for all estimated parameters together, although it was noted before that the correlations between parameters for the $X, Y$ and $Z$ axes are uncorrelated.

Based on a uniform observation weight of $1.5 \times$ $10^{-8} \mathrm{~m} / \mathrm{s}^{2}$, obtained by multiplying the upper level of the angular acceleration reconstruction error (see Sect. 3.1) with the arm length of 0.5 , the formal error is about $1 \mathrm{~nm} / \mathrm{s}^{2}$ for the relative biases and $2 \times 10^{-5} \mathrm{~nm} / \mathrm{s}^{3}$ for the relative bias drifts. The formal errors for the scale factors range between 0.011 and 0.038 for the $X$ axis, 0.0079 and 0.0084 for the $Y$ axis, and 0.0041 and 0.0070 for the $Z$ axis. These error levels are of the same order of magnitude as the estimated deviations from 1 for the scale factors.

\section{Conclusions}

A calibration method has been proposed for the six individual accelerometers of the GOCE gradiometer that predominantly relies on the availability of high-quality star-tracker observations. The method requires the use of an a priori gravity field model, but it was found that the effect of errors of state-of-the-art or even older generation gravity field model errors is negligible (for example, the differences between the EGM96 and JGM3 gravity fiel models are much larger than the claimed accuracy of the most recent global gravity models, such as those including data from GRACE (Reigber et al. 2005). It was found that with this method scale factors can be obtained with an accuracy of $0.01-0.02$ or better based on a test data set of end-to-end observations, where all anticipated error sources were modeled and taken into account. Such an accuracy level is; however, a few orders of magnitude worse than the level aimed at for GOCE (Cesare 2005). Therefore, the method outlined in this paper should be considered to serve as a possible additional check of the accelerometer observations. All the results are based on the assumption that observations of only one star-tracker are nominally available, where the bore sight is rotated by $60^{\circ}$ with respect to the gradiometer reference frame. In reality, improvements might be obtained by combining observations of two star-trackers that are always observing on board of GOCE. 
For obtaining the best accelerometer scale factor values, it is required to use an averaging window in the estimation of angular rotation rates and accelerations from the star tracker observations in order to reduce their noise (typically of the order of $\left.10-50^{\prime \prime}\right)$. In addition, it can be concluded that it is required to apply the same averaging window to the accelerometer observation equations. Finally, best results were obtained by including the estimation of accelerometer bias drifts.

Acknowledgments The European Space Agency is acknowledged for sponsoring the work that led to the study described in this paper and for providing the end-to-end data which was produced by a detailed simulator built by Alenia and many of its sub-contractors.

Open Access This article is distributed under the terms of the Creative Commons Attribution Noncommercial License which permits any noncommercial use, distribution, and reproduction in any medium, provided the original author(s) and source are credited.

\section{References}

Alenia (1999) GOCE-Gravity field and ocean circulation explorer, Phase A, Summary Report, ESA Contract 13028/98/NL/GD, Alenia Aerospazio, June

Bouman J, Koop R, Tscherning C, Visser P (2004) Calibration of GOCE SGG data using high-low SST, terrestrial gravity data, and global gravity field models. J Geod 78(1-2):124-137. doi:10.1007/ s00190-004-0382-5

Bruinsma S, Biancale R (2003) Total densities derived from accelerometer data. J Spacecraft Rockets 40(2): 230-236

Bruinsma S, Tamagnan D, Biancale R (2004) Atmospheric densities derived from CHAMP/STAR accelerometer observations. Planet Space Sci 52(4):297-312. doi:10.1016/j.pss.2003.11.004
Catastini G, Cesare S, De Sanctis S, Dumontel M, Parisch M, Sechi G (2006) Predictions of the GOCE in-flight performances with the end-to-end system simulator. In: 3rd GOCE User Workshop, 6-8 November 2006, Frascati, Italy. ESA SP-627, pp 9-16

Cesare S (2005) Performance requirements and budgets for the gradiometric mission. Technical Note, GOC-TN-AI-0027, Issue 3, Alenia Spazio, Turin, May

Cesara C, Catastini G (2005) Gradiometer on-orbit calibration procedure analysis. Technical Note to ESA, GO-TN-AI-0069, Issue 3, Alenia Aerospazio, May

Drinkwater M, Haagmans R, Muzzi D, Popescu A, Floberghagen R, Kern M, Fehringer M (2007) The GOCE gravity mission: ESA's first core explorer. In: 3rd GOCE user workshop, 6-8 November 2006, Frascati, Italy. ESA SP-627, pp 1-7

ESA (1999) Gravity field and steady-state ocean circulation mission. Reports for Mission Selection, The Four Candidate Earth Explorer Core Missions, SP-1233(1), European Space Agency, July

Lemoine FG, Kenyon SC, Factor JK, Trimmer RG, Pavlis NK, Chin DS, Cox CM, Klosko SM, Lutchke SB, Torrence MH, Wang YM, Williamson RG, Pavlis EC, Rapp RH, Olsen TR (1998) The Development of the Joint NASA GSFC and the National Imagery and Mapping Agency (NIMA) Geopotential Model EGM96, NASA/TP-1998-206861

Montenbruck O, Gill E (2000) Satellite Orbits-Models methods applications. Springer, Heidelberg ISBN 3-540-67280-X

Reigber C, Schmidt R, Flechtner F, Konig R, Meyer U, Neumayer KH, Schwintzer P, Zhu SY (2005) An Earth gravity field model complete to degree and order 150 from GRACE: EIGEN-GRACE02S. J Geodyn 39(1): 1-10

Tapley BD, Watkins MM, Ries JC, Davis GW, Eanes RJ, Poole SR, Rim HJ, Schutz BE, Shum CK, Nerem RS, Lerch FJ, Marshall JA, Klosko SM, Pavlis NK, Williamson RG (1996) The Joint Gravity Model 3. J Geophys Res 101(B12): 28,029-28,049

Tapley BD, Bettadpur S, Watkins M, Reigber C (2004) The gravity recovery and climate experiment experiment, mission overview and early results. Geophys Res Lett 31:L09607. doi:10.1029/ 2004GL019920

Visser PNAM (2007b) GOCE gradiometer validation by GPS. Adv Space Res 39(10):1630-1637. doi:10.1016/j.asr.2006.09.014 